\title{
Tendinopatía de De Quervain (2). Resultado del tratamiento quirúrgico con reconstrucción del retináculo del primer compartimento extensor
}

\author{
G. Celester Barreiro ${ }^{(1)}$, F. Cano Obregón ${ }^{(1)}$, A. Castro Río ${ }^{(2)}$, \\ J. M. Rodríguez VÁzQuez ${ }^{(2)}$
}

Servicio de Cirugía de la Mano del Hospital Modelo. Mutua La Fraternidad. A Coruña. (1) ESPECIALISTA EN CIRUGÍA ORTOPÉDICA Y TRAUMATOLOGÍA

(2) MEDICINA ASISTENCIAL

Correspondencia:

Dr. Gabriel Celester Barreiro

Avda. del Ejército 12, bajo (Mutua)

15006 A Coruña. Telf. 981296588

e-mail: gcelester@fraternidad.com

\begin{abstract}
Objetivo: Analizar los resultados del tratamiento quirúrgico de la tendinopatía de De Quervain con dos técnicas diferentes: la habitual, de apertura retinacular simple del primer compartimento extensor (grupo A: 27 casos en 26 pacientes. 1 caso bilateral) y una técnica reconstructora de la polea (grupo B: 48 pacientes).
\end{abstract}

Material y método: Se estudiaron 253 muñecas diagnosticadas consecutivamente de Tendinopatía de De Quervain en 252 pacientes (1 caso bilateral). De ellas, $75(30 \%)$ fueron tratados quirúrgicamente. En 27 casos se procedió a la apertura simple del primer compartimento (Grupo A), mientras que en los 48 casos restantes se les practicó la reconstrucción del mismo (Grupo B).

En 7 pacientes del grupo A la Tendinopatía de De Quervain se asociaba a Síndrome de Intersección (26\%). En el grupo B esta asociación se dio en 11 casos $(23 \%)$. En todos ellos, el Síndrome de Intersección fue intervenido en el mismo acto quirúrgico.

Resultados: Todos los pacientes curaron con el tratamiento quirúrgico, excepto una paciente (con mucha probabilidad, una simuladora rentista). El período de baja laboral fue de 88,1 días en el grupo A, y de 53 días en el grupo B. Las complicaciones
Purpose: To analyse the results of the surgical treatment of the De Quervain's disease, between two different methods: the common one, simple release of the First Extensor Compartment retinaculum (A Group: 27 cases in 26 patients, 1bilateral case) compared to a renovative technique of the extensor retinaculum (B group: 48 patients).

Material and methods: 253 wrists consecutively diagnosed of De Quervain's disease were studied in 252 patients, 75 out of them were treated surgically $(30 \%)$. We proceded with simple release of first compartment in 27 cases (group A), while we reconstructed such compartment in 48 of the rest of the cases (group B).

The Intersection Syndrome (tenosynovitis of the radial wrist extensors) was asociated to De Quervain's desease in 7 patients of $\mathbf{A}$ group (26\%) and 11 cases of $\mathbf{B}$ group (23\%). In all of them, this Intersection Syndrome was operated in the same surgery.

Results: All of patients were cured with surgical treatment, except a female patient (very probably she was pretending to have an illness to earn an economic compensation). The work disability days were 88.1 days in $\mathbf{A}$ group and 53 days in $\mathbf{B}$ group. A group complications included 2 abductor polli- 
encontradas en el grupo A fueron 2 casos de luxación del abductor pollicis longus y una sección del abductor pollicis brevis; la persistencia de dolor a la palpación y prueba de Finkelstein positiva fueron mayores de 2,5 meses en 9 casos. En el grupo B hubo dos lesiones nerviosas iatrogénicas, ningún caso de luxación y la prueba de Finkelstein se negativizó siempre antes de los 2 meses.

Conclusiones: La apertura longitudinal simple del primer compartimento extensor del radio puede provocar la luxación del abductor pollicis longus fuera de su lecho óseo en los movimientos de flexión de la muñeca combinada con abducción del pulgar. La técnica reconstructora de la polea evita esta complicación.

La sintomatología tras la intervención quirúrgica desaparece antes con la técnica reconstructora, y es más precoz la reincorporación laboral.

Es frecuente la asociación de tendinopatía de De Quervain con otros procesos de etiología ocupacional. En esta serie se operaron 29 procesos asociados en 28 pacientes (37\% del total). La más común es con el Síndrome de Intersección, que se dio en el $24 \%$ de los pacientes operados en esta serie.

Palabras clave: enfermedad de De Quervain, tendinopatía estenosante de De Quervain, síndrome de intersección. cis longus luxation and an abductor pollicis brevis section; tenderness and Finkelstein test positive took more time than 2.5 months in 9 cases. B group complications included two yatrogenic nervous injuries, none any luxation cases and Finkelstein test was negative before two months.

Conclusions: Simple release of the First Extensor Compartment retinaculum of the wrist may cause a luxation of the abductor pollicis longus out off its bone bed in wrist flexion movements with thumb abduction. This technique of restoration the extensor retinaculum avoids this complication.

Symptoms after surgery dessapear earlier with a technique of restoration, and reincorporation to work is also quicker.

De Quervain's disease is frecuently asociated with another process of work ethiology. 29 associated processes in 28 patients were operated in this study $(37 \%)$. The most frecuent patology was the Intersection Syndrome which was present in the $24 \%$ of the patients who underwent surgery.

Key words: De Quervain's disease, De Quervain's stenosing tendovaginitis, intersection syndrome.

\section{INTRODUCCIÓN}

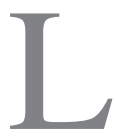

a afectación del extensor pollicis brevis (EPB) y del abductor pollicis longus (APL) en el primer compartimento extensor de la muñeca se conoce con el epónimo del cirujano suizo Fritz de Quervain desde la publicación de su artículo ${ }^{1}$ en 1895.

Existe un acuerdo unánime de todos los autores en que el tratamiento inicial debe ser conservador mediante reposo, antiinflamatorios, inmovilización con férulas, infiltración de corticoides y rehabilitación. Sólo cuando éste fracasa, lo que ocurre entre el 40 y el $70 \%$ de los $\operatorname{casos}^{2}$, estaría indicado el tratamiento quirúrgico.

La técnica habitual consiste en la apertura longitudinal del retináculo por su borde más dorsal para evitar la luxación tendinosa. No obstan- te, tras esta única medida puede producirse una luxación de los tendones, sobre todo la del $a b$ ductor pollicis longus en los movimientos conjugados de abducción completa del pulgar con flexión máxima de la muñeca, con la consiguiente persistencia de los síntomas preoperatorios. Para evitar esta complicación se ha propuesto la reconstrucción de la polea mediante diversas técnicas.

Codega ${ }^{3}$ reconstruye el retináculo con dos bandeletas obtenidas de la propia polea una vez resecada. Kapandji ${ }^{4}$ propone la apertura oblicua de la polea obteniendo dos bandeletas triangulares que sutura uniendo los dos vértices libres. Le Viet $^{5}$ realiza la sutura de la bandeleta radial de la polea al tejido celular subcutáneo para hacer un muro que impida el desplazamiento volar de los tendones. Littler et al. ${ }^{6}$ suturan la polea, una 
vez abierta, dejando fuera el EPB y Ramesh y Britton $^{7}$ confeccionan un nuevo retináculo con una bandeleta del ligamento anular dorsal del carpo.

\section{OBJETIVO}

El objetivo de este trabajo es analizar los resultados del tratamiento quirúrgico tras la técnica clásica de apertura simple, y los obtenidos tras la reconstrucción de la polea del primer compartimento extensor.

\section{MATERIAL Y MÉTODO}

En este estudio de cohortes, retrospectivo y no aleatorizado, se analizaron 253 muñecas diagnosticadas consecutivamente de tendinopatía de De Quervain (TDQ), de las que 75 (30\%) precisaron tratamiento quirúrgico.

Se dividieron en dos series:

Grupo A (técnica de apertura simple de la polea): 27 casos en 26 pacientes ( 1 caso bilateral)

Grupo B (técnica reconstructora de la polea): 48 pacientes.

Con motivo de dos reintervenciones por luxación del abductor pollicis longus, todos del grupo A, desde octubre de 2003 se realiza sistemáticamente una técnica de reconstrucción de la polea. Todos los pacientes diagnosticados por primera vez recibieron tratamiento conservador con AINES, inmovilización con férula tipo
EMO y fisioterapia. A los que llegaron ya diagnosticados y tratados sin éxito con largo tiempo de evolución, se les indicó la intervención quirúrgica. De los pacientes operados, 9 habían sido infiltrados con corticoides.

Edad: La media de edad fue de 41,7 años. En el grupo $\mathbf{A}$ la media fue de 35,5 años $(\mathrm{r}=22-67)$. En el grupo B la media fue de 44 años $(r=26-$ 87).

Género: 64 mujeres (86\%), 10 hombres (14\%). En el grupo A había 23 mujeres y 3 hombres; en el grupo B, 41 mujeres y 7 hombres.

Lado: Derecho afectado en 50 manos; izquierdo en 25. En el grupo A se afectó la mano derecha en 19 casos y la izquierda en 8. En el grupo $\mathbf{B}$ la derecha estaba afectada en 31 pacientes y la izquierda en 17; en este segundo grupo había dos mujeres zurdas en las que se afectó la mano dominante.

Patología asociada: En total se operaron 28 entidades concomitantes: 18 síndromes de intersección (SI), 4 síndrome del túnel carpiano, 3 rizartrosis del pulgar, 2 tenosinovitis estenosante de algún dedo y 1 epicondilitis. El SI resultó la asociación más frecuente (24\% de las muñecas): 7 del grupo A, 11 en el grupo $\mathbf{B}$.

Técnica quirúrgica: Cuando no existía un SI concomitante, que requiere siempre una mayor exposición quirúrgica para liberar las adherencias del EPB con la sinovial del extensor carpi radialis longus y extensor carpi radialis brevis, se realizó una incisión transversal de unos 2 ó $3 \mathrm{~cm}$ sobre la estiloides radial, lo que permite un suficiente abordaje y deja una cicatriz más
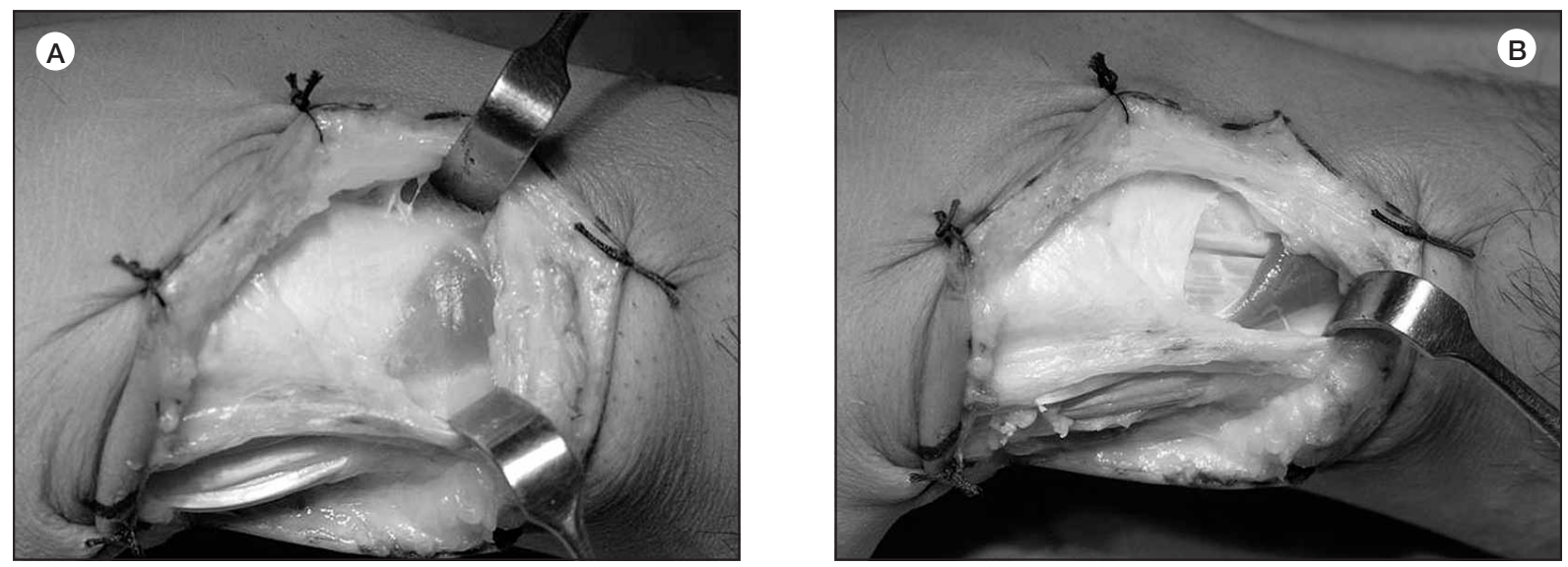

Figura 1: A: Síndrome de intersección. Nótese la adherencia del EPB a la sinovial del $2^{\circ}$ compartimento. B: Liberada esa adherencia, se conserva la parte distal de la polea de este compartimento. 


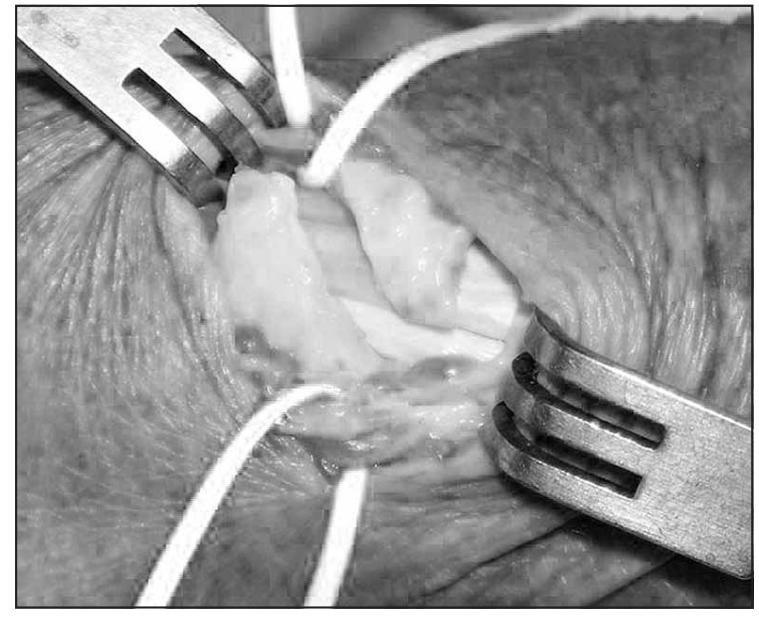

Figura 2: Apertura de la polea en $S$ itálica en los primeros casos de esta serie.

estética que las incisiones longitudinales o en zeta. En los casos de coexistencia con un SI la incisión fue oblicua o en S itálica, desde la línea media del antebrazo en su cara dorsal, a unos 3,5 $\mathrm{cm}$ de la interlínea, hasta el borde anterior de la apófisis estiloides del radio.

En los casos con SI asociado se escindieron las adherencias del EPB con los extensores radiales de la muñeca, se realizó la sinovectomía de éstos y se conservó la parte distal de la polea del $2^{\circ}$ compartimento extensor (Figuras 1A y 1B).

Todos fueron operados con manguito de isquemia. En los primeros casos se dejaba un drenaje no aspirativo. Posteriormente se abandonó al apreciar que no suele ser preciso, así como el empleo de coagulación térmica, que no sólo es innecesario si se respetan los vasos que acompañan a las ramas del nervio radial sino que tiene el inconveniente de que puede lesionar alguna de éstas.

En todos los casos se realizó una escisión de la membrana sinovial, que es imprescindible para lograr ver los tendones, sobre todo en la parte distal del primer compartimento.

En el grupo A se realizó una apertura longitudinal de la polea por su parte dorsal o central, conservando siempre la parte más volar de la misma para evitar la luxación tendinosa.

En el grupo $\mathbf{B}$ se realizaron dos tipos de apertura de la polea. En los primeros pacientes se efectuó una incisión en S itálica (Figura 2) y se suturó el vértice de los colgajos resultantes

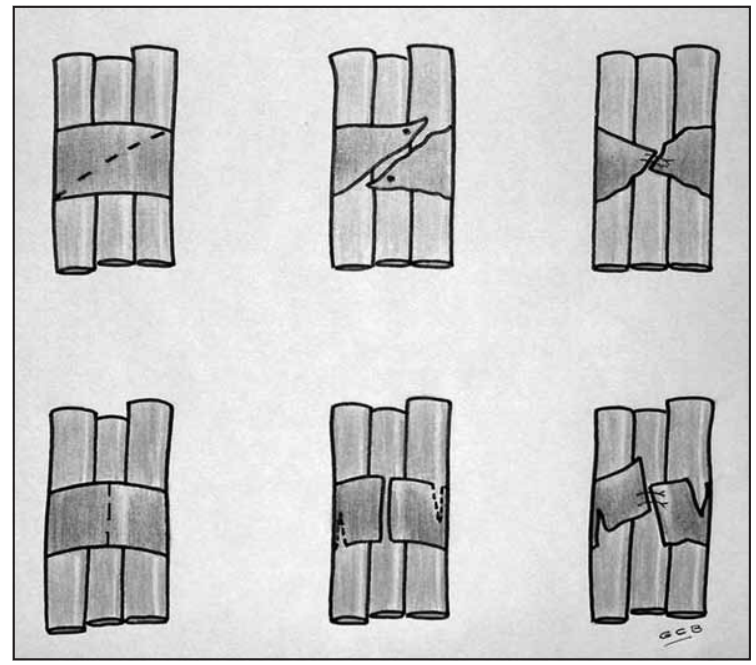

Figura 3: Dibujo de ambas modalidades de la técnica reconstructora. Arriba: Apertura oblicua y sutura de los bordes. Abajo: Apertura longitudinal y sendas incisiones en la base de cada colgajo.

(Figura 3, parte superior). En los siguientes, por la dificultad que representa la disección sobre el tabique, se hizo una longitudinal efectuando sendas incisiones parciales en la base de cada colgajo (Figura 3, parte inferior). Ambas formas de reconstrucción tienen como finalidad aportar una polea más corta, pero más amplia que la previa, para eliminar la sintomatología y evitar la luxación tendinosa. Las dos formas, diseñadas antes de conocer la publicación de Kapandji ${ }^{4}$, son muy semejantes a las descritas por este autor, pero la segunda, de dos incisiones parciales tras la apertura longitudinal y no oblicua como hace Kapandji ${ }^{4}$, facilita mucho la técnica, en la cual, dependiendo del ancho resultante de ambos colgajos, se da uno, dos o tres puntos (Figura 4: A, B y C), casi siempre dos.

En ambos grupos se resecó el tabique entre el EPB y el APL para aportar mayor espacio a los tendones; en los dos se empleó anestesia regional - casi siempre axilar-y sólo en una paciente se usó anestesia general para operarle también un espolón calcáneo. En todos se utilizó manguito de isquemia y se suturó la piel con nylon monofilamento de 4/0. Habitualmente no se sutura el subcutáneo para evitar material extraño, fuente de posible fibrosis. Se colocó un vendaje compresivo (en ningún caso férula) y se permitió a los pacientes que moviesen libremente el pulgar evitando las pinzas de fuerza. 

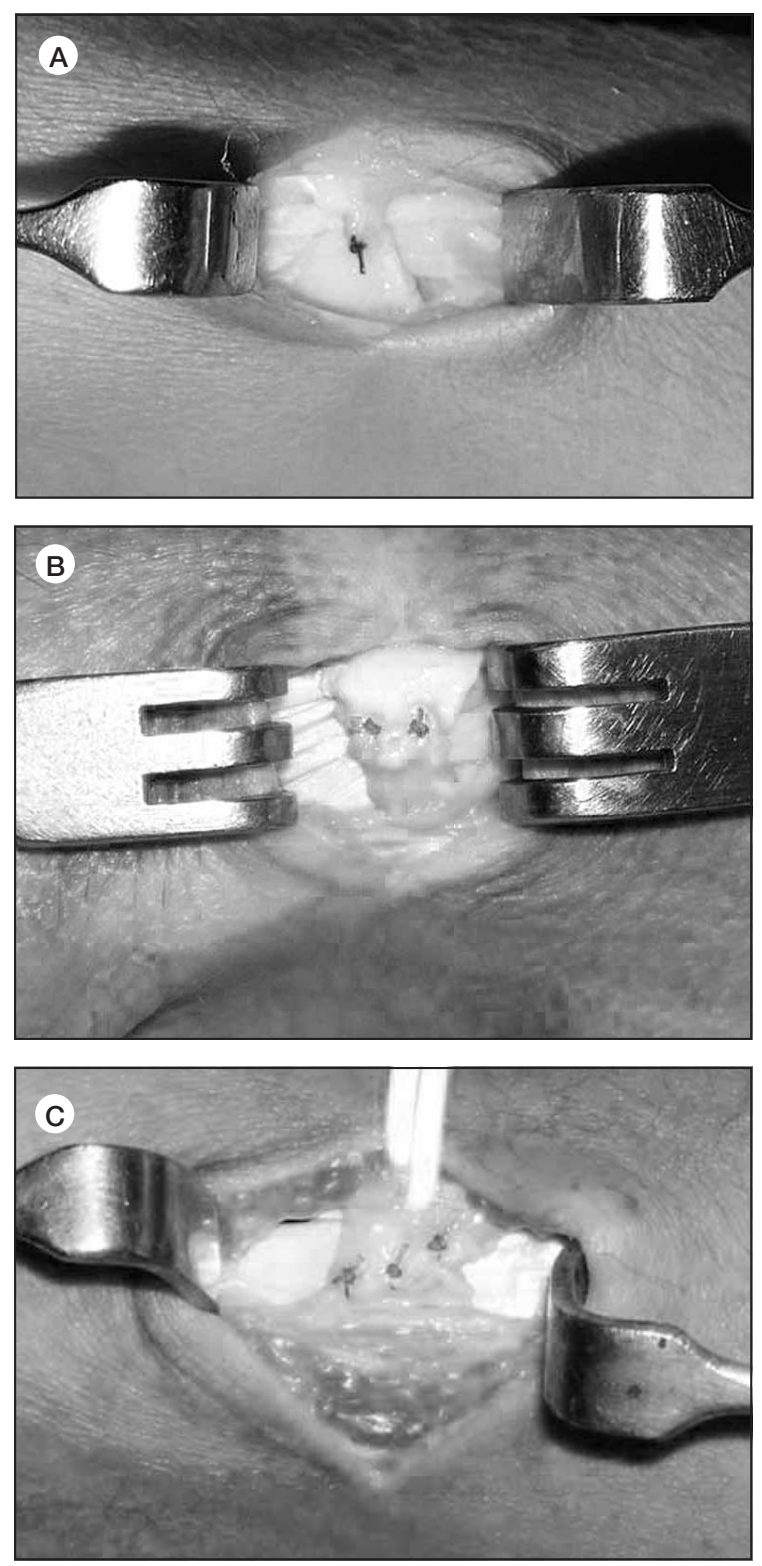

Figura 4: A: Polea estrecha: sutura con un punto. B: Polea mediana: sutura con dos puntos. C: Polea ancha: sutura con tres puntos.

En 4 pacientes que presentaban un gran volumen tendinoso se reconstruyó la polea según la técnica de Kapandji ${ }^{4}$, pero dejando fuera el tendón del EPB como aconsejan Littler y Freed$\operatorname{man}^{6}$ (Figura 5).

\section{RESULTADOS}

Todos los pacientes operados curaron y se reincorporaron a su actividad anterior, excepto una paciente que se negó a aceptar el parte de alta. A esta paciente, de quien sospechábamos que nos engañaba y nos lo confirmó una vecina, también paciente, al decirnos que cultivaba sin ninguna dificultad su huerto, se le puso un detective porque solicitó una incapacidad permanente total. Las fotografías y el vídeo la sorprendieron conduciendo su automóvil, realizando la compra en un mercado y realizando pinzas de fuerza absolutamente normales con el pulgar. Pese a todo, ganó el juicio y no se reincorporó.

Tiempo de baja laboral: el período de baja laboral - que de algún modo indica el tiempo de la desaparición sintomatológica-, se contabilizó a partir del día de la intervención, excluyendo por tanto el período de tratamiento previo a la cirugía. Para medir el tiempo de reincorporación a las actividades habituales sin dolor se excluyó un paciente al que se causó la lesión de una rama sensitiva del nervio radial, a todos aquéllos en los que se realizó alguna otra técnica concomitante, y a una paciente (delegada comercial) que no quiso coger días de permiso. Se analizó aparte los que tenían un síndrome de intersección concomitante.

La media global fue de 65 días de baja. En el grupo A la media resultó de 88,1 días; en el grupo $\mathbf{B}$ de 53 días.

Como en el grupo A era mayor la coexistencia de patología asociada que en el grupo B, cabe pensar en un pequeño sesgo con respecto a los días de baja, pero también es cierto que esa patología (STC o dedos en resorte, por ejemplo) curan al mismo tiempo que la TDQ.

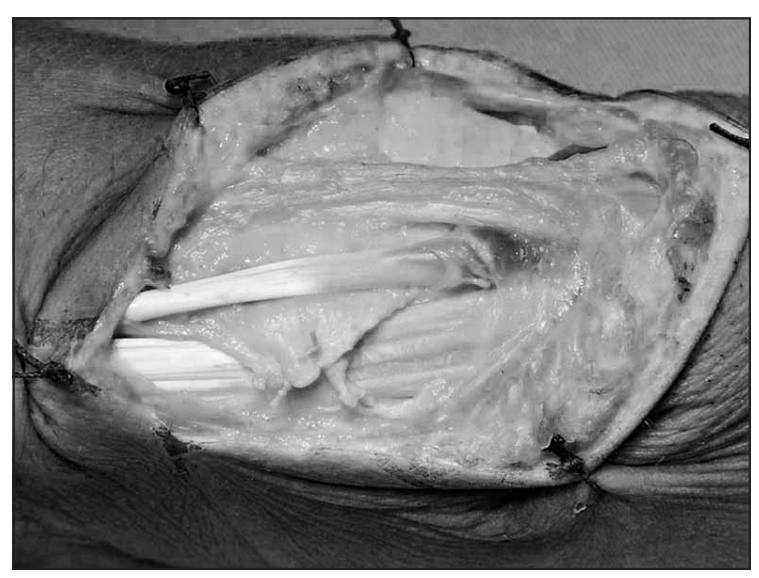

Figura 5: En algún caso con gran volumen tendinoso se reconstruyó la polea dejando fuera el APB. 
Complicaciones: En los pacientes a los que se realizó apertura simple sin reconstrucción de la polea hubo 2 casos de luxación dolorosa del APL al realizar la abducción del pulgar con la muñeca en flexión. Ambos casos (las dos eran charcuteras) fueron reintervenidos para reconstruirle la polea. A una paciente se le seccionó accidentalmente el EPB y no se suturó (la pérdida de este tendón no deja ninguna secuela significativa; de hecho, el EPB falta entre el 5\% y el 7\% de las personas ${ }^{8,9}$. Otras 2 pacientes tuvieron una persistencia temporal de los síntomas, que fueron cediendo en pocas semanas con fisioterapia.

En 2 casos del grupo B hubo complicaciones neurológicas; una paciente tuvo una prolongada hipoestesia de una rama sensitiva del nervio radial (quizás por una tracción excesiva de un baseloop) que se recuperó espontáneamente. En un paciente se lesionó parcialmente una rama nerviosa por contacto con el bisturí eléctrico al coagular un vaso. En el mismo acto quirúrgico se resecó la parte lesionada y se realizó neurorrafia. Este paciente cursó con una causalgia e interpuso una demanda judicial.

En el grupo A la prueba de Finkelstein perduró más de 2,5 meses en 9 casos. En el grupo B esta prueba se negativizó siempre antes de los 2 meses. Curiosamente, tras la reconstrucción de la polea el punto más doloroso a la palpación no se encontró sobre el retináculo, como en el período preoperatorio, sino a $1 \mathrm{~cm}$ de la estiloides, al hacer presión sobre los tendones en la oquedad del borde radial del carpo.

\section{DISCUSIÓN}

De 253 casos de TDQ tratados inicialmente de forma conservadora, fueron operadas 75 manos (el 30\%); es decir, 1 de cada 3 pacientes. Cifras que muestran un índice elevado de fracasos con el tratamiento conservador ante este tipo de patología (para Umlas ${ }^{2}$ curan con tratamiento conservador del $30 \%$ al $60 \%$ de los casos), fracaso que en esta serie sin duda guarda relación con el ámbito laboral en el que fueron vistos una gran parte de los pacientes.

Edad: La media global de 41,7 años es muy semejante a la de otras series publicadas (38 en la de Arroyo et al. ${ }^{10}$ y 42,6 en la de Wetterkamp et al. $\left.{ }^{11}\right)$, aunque la edad, lógicamente, depende mucho de la población estudiada. Rossi ${ }^{12}$ encuentra la mayor incidencia en mujeres de 20 a 40 años en un grupo de jugadoras de volleyball, y Wolf et al. ${ }^{13}$ registran el mayor porcentaje en mujeres mayores de 40 tras estudiar una amplia población de personal militar.

Género: Con el $86 \%$, es evidente la mayor afectación de TDQ en mujeres (casi 9 de cada 10 pacientes), lo que coincide con la bibliografía revisada. En esta serie se encontró una mayor predominancia que en otras $(70 \%$ en la Wetterkamp y $75 \%$ en la de Arroyo) probablemente por el tipo de población laboral del que provienen la mayor parte de las pacientes, una mutua de accidentes con muchas empresas aseguradas de la industria textil, así como supermercados en los que predomina el personal femenino: carniceras, charcuteras, pescaderas, fruteras, panaderas y cajeras-reponedoras.

Lado: En 50 manos estaba afectado el lado derecho $(66,7 \%)$ y en 25 el izquierdo $(33,3 \%)$. En dos mujeres zurdas la mano izquierda fue la afectada, por lo que la enfermedad en el lado dominante se dio en el 69,3\% de los casos. La predominancia del lado dominante resultó idéntica a la serie de Arroyo et al. ${ }^{10}$, quienes también hallaron un $69 \%$ de pacientes con afectación del lado derecho.

Tipo de incisión cutánea: Como ya se ha dicho, en todos los casos en que no había un SI asociado se realizó una incisión transversal. La mayoría de autores así lo aconsejan por razones estéticas y para evitar cicatrices dolorosas ${ }^{8,9}$, si bien algunos emplean siempre la longitudina ${ }^{10} \mathrm{y}$ otros alternan ambas vías ${ }^{14} \mathrm{o}$ utilizan incisiones en zeta ${ }^{15}$.

Forma de la nueva polea: La polea reconstruida resulta siempre más corta que la previa pero más amplia (Figuras 6A y 6B). Con la técnica de Codega $^{3}$, algo más dificultosa técnicamente y que exige más suturas (cuatro en lugar de una), con lo que pueden aumentar las complicaciones, se obtiene una polea más larga pero incompleta al faltarle el techo en su zona media. En cualquier caso, ambas técnicas cumplen la misma función y seguramente no habrá entre ellas diferentes resultados.

Patologías asociadas: Por su carácter ocupacional, es muy frecuente la asociación de TDQ 
con otras patologías del miembro superior del mismo origen. Se ha referido su concomitancia con diversas entidades, especialmente con dedos en resorte, síndrome del túnel carpiano (STC), epicondilitis, bursitis y tendinitis del hombro. Arroyo et al. ${ }^{10}$ encontraron patología asociada en el $17 \%$, Wetterkamp et al. ${ }^{11}$ en el $32,8 \%$ y Arons ${ }^{16}$ refiere 23 diagnósticos asociados en 16 pacientes operados.

En esta serie se operó patología concomitante del mismo miembro en 12 pacientes del grupo A (7 SI, 3 STC, 1 epicondilalgia, 1 tenosinovitis estenosante digital) y en 16 del grupo B (11 SI, 1 STC, 3 rizartrosis del pulgar y 1 tenosinovitis estenosante digital). En total, 28 pacientes ( $37,3 \%$ del total) fueron operados de 29 procesos asociados.

Anomalías anatómicas: Se han apreciado alteraciones anatómicas en 4 manos $(5,6 \%)$ : en 3 entraban los vientres musculares dentro del túnel y una paciente tenía una polea anormalmente larga. Algunos autores ${ }^{14}$ señalan la existencia de anomalías hasta en el 18,3\% de los casos.

Tratamiento previo con infiltraciones: Casi todos los autores aconsejan el tratamiento con infiltraciones de corticoides pero pocos reflejan cuantos pacientes infiltrados acaban siendo intervenidos. Para Wolfe ${ }^{9}$ la inmovilización con férula sola produjo un fracaso del $70 \%$ en 37 muñecas y no proporcionó beneficio adicional cuando se combinó con la inyección de corticoides en la vaina, mientras que en una reciente revisión bibliográfica, Ilyas ${ }^{17}$ encuentra que más del $70 \%$ de los pacientes curan con tratamiento conservador,

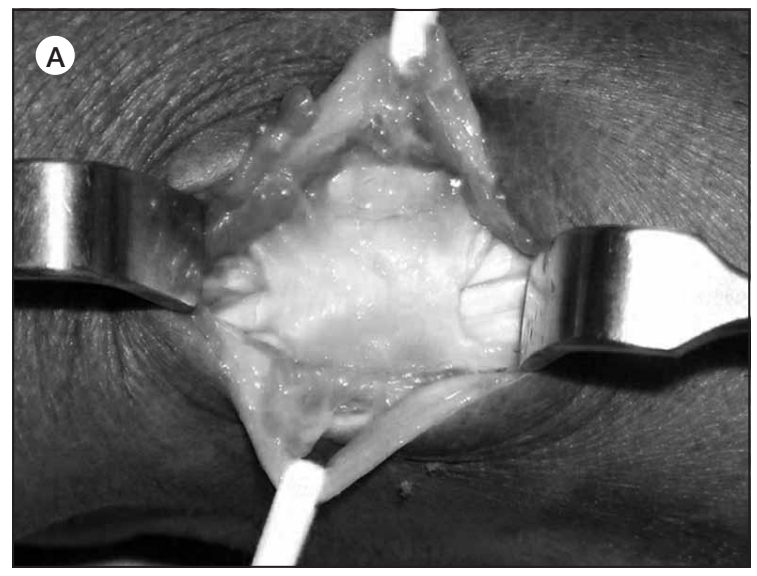

especialmente con la inyección local de corticoides: McKenzie ${ }^{18}: 93 \%$, Harvey et al ${ }^{19}: 82 \%$, Witt et $\mathrm{al}^{20}: 62 \%$, Anderson et $\mathrm{al}^{21}: 90 \%$.

Tratamiento postoperatorio: En todos los casos se empleó un vendaje compresivo permitiendo la movilidad activa de la muñeca y el pulgar desde el primer momento, restringiendo las pinzas de fuerza durante las primeras semanas. La mayoría de autores así lo aconsejan ${ }^{10,11}$, si bien algunos emplean una férula durante 2 ó 3 semanas ${ }^{8}$ y otros sólo cuando consideran que los tendones liberados han podido quedar inestables tras la simple apertura de la polea ${ }^{9}$.

Sólo en los pacientes de peor evolución se prescribe rehabilitación ulterior. No suele ser precisa ya que al no usar férulas se previenen las adherencias y con ello la rigidez de la muñeca. Por otro lado, los masajes y maniobras forzadas del fisioterapeuta pueden irritar las ramas sensitivas del nervio radial. En esta serie se indicó fisioterapia a menos del $20 \%$ de los pacientes

Tiempo de baja laboral: En la mayoría de los casos se encontró una relación clara entre la entidad patológica y el tipo de actividad. De los 75 pacientes operados, 7 estaban jubiladas (todas mujeres) y 68 en edad laboral; de los cuales 64 estaban en activo y 4 no tenían ningún trabajo remunerado. Cincuenta y un pacientes ejercían profesiones de fuerza y 13 tenían labores manuales de pequeños esfuerzos (administrativas, maestras, comerciales), aunque la mayoría de ellas ejercían también de amas de casa.

La media de días de baja, incluyendo a todos los pacientes - trabajadores y parados y con

Figura 6, A y B: Polea menos larga pero más espaciosa.

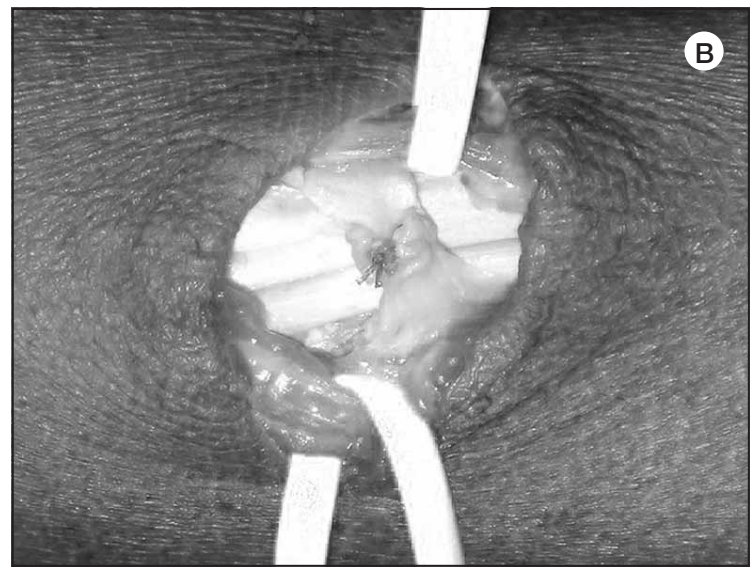


o sin reconstrucción de la polea-, fue de 65 días. Pero hubo una manifiesta diferencia en el tiempo de baja de los pacientes de la mutua con respecto a los autónomos y trabajadores que por diversos motivos no se benefician de una baja laboral prolongada. Los de la mutua tardaron 21 días más de media en reincorporarse a sus ocupaciones.

Existen pocas series que reflejen el período de absentismo laboral. Estudiando sólo una población laboral, Arroyo et al. ${ }^{10}$ muestran unas cifras muy similares a las obtenidas en el grupo A de esta serie, en tiempos que oscilan de 21 a 330 días, con una media de 97.

El tiempo de baja aumentó en los pacientes operados de patologías concomitantes. En aquellos que tenían un Sí asociado la media fue de 27,7 días más.

Complicaciones: Todos los autores reflejan frecuentes complicaciones en el tratamiento quirúrgico de la TDQ, hasta el punto de que Arons ${ }^{16}$ registra 14 complicaciones en 16 pacientes. Las más comunes son la persistencia del dolor (entre el $18 \%$ y el $\left.25 \%{ }^{10,11}\right)$, disestesias sobre la cicatriz (entre el 3\% y el $12 \%{ }^{10,11}$ ), lesiones del nervio radial y subluxación del APL.

En esta serie se registraron complicaciones en 7 pacientes, 3 de ellas de origen iatrogénico (una sección del EPB y dos neurológicas).

Reintervenciones: No son raras las reintervenciones tras el tratamiento quirúrgico de la TDQ. El 13\% de los pacientes de Arroyo et al. ${ }^{10}$ fueron reoperados por diversos motivos, y el $3,4 \%$ de los pacientes de Wetterkamp et al. ${ }^{11}$ lo precisaron por neurolisis ( 3 casos) o por queloide de la cicatriz (1 caso).

Una causa de reintervención es la luxación del APL. En esta serie se apreció una subluxación en el $18 \%$ de los pacientes intervenidos con la destechadura simple. La mayoría curaron espontáneamente sin una nueva intervención, pero 2 pacientes tuvieron que ser reoperadas por este motivo, reconstruyéndoseles la polea en la segunda intervención.
Aspectos legales: En el ámbito laboral no son infrecuentes las demandas y solicitudes de incapacidad tras el tratamiento quirúrgico de la TDQ.

En la serie de Arroyo et al. ${ }^{10}$ de 93 pacientes el $13 \%$ fueron revisados por el tribunal médico de valoración de incapacidades. Dos resultaron con incapacidad permanente total, 2 con incapacidad permanente parcial y 2 obtuvieron un baremo por secuelas no incapacitantes. En esta serie hubo una incapacidad permanente total en una clara simuladora. El paciente al que lesionamos una rama sensitiva del nervio radial está pendiente de juicio.

\section{CONCLUSIONES}

1. Se constata que con la reconstrucción de la polea no se produce la luxación del APL, complicación que obliga a reintervenciones en algunos pacientes a los que se le realiza una simple apertura del compartimento.

2. El tiempo de recuperación y de reincorporación laboral es mucho menor en los pacientes a los que se reconstruye el retináculo.

3. Es frecuente la asociación de TDQ con otros procesos de etiología ocupacional. La más común es con el SI, que se dio en el $24 \%$ de los pacientes operados en esta serie.

4. Al margen de otros factores coyunturales (embarazo, tratamiento con fluoroquinolonas, etc.) o anatómicos que predisponen a padecer la enfermedad (como el número elevado de tendones accesorios del APL, o la presencia de vientres musculares dentro del retináculo), la TDQ tiene una clara relación con el tipo de actividad laboral.

5. El alto porcentaje de pacientes con recidivas tras el tratamiento conservador y que necesitan tratamiento quirúrgico (1 de cada 3 ) probablemente se deba a que una vez mejorados, casi todos los trabajadores se reincorporan a la misma actividad que produjo la enfermedad. 


\section{BIBLIOGRAFÍA}

1. De Quervain F. Ueber eine form von chronischer tendovaginitis. Korrespondenz-Blatt Schweizer Aerzrte 1895, 25: 389-94. (On a form of chronic tendovaginitis). Translated article in $\mathrm{J}$ Hand Surg B, 2005; 30: 388-91.

2. Umlas $M E$ y Gelberman $\mathrm{RH}$. Tratamiento quirúrgico de la enfermedad de de Quervain. En: Gelberman RH (Ed). Muñeca. Madrid: Ed Marbán, 1999: 383-92.

3. Codega G. Tecnica chirurgica nella malatia di De Quervain. La Patologia del Polso. Padoue: Piccin Nuova Libraria, 1987: 203-7.

4. Kapandji Al. Plastie d'agrandissement de la coulisse radiostyloïdienne dans le traitement de la ténosynovite de De Quervain. Ann Chir Main, 1990, 1: 42-46.

5. Le Viet D. Ténosynovite de De Quervain. Maîtrise Orthopédique 2002; 114: 10-1.

6. Littler JW, Freedman DM, Malerich MM. Compartment reconstruction for De Quervain disease. J. Hand Surg B, 2002; 27: 242-4.

7. Ramesh R, Britton JM. A retinacular sling for subluxing tendons of the first extensor compartment. J. Bone Joint Surg B, 2000; 82: 424-5.
8. Phalen GS. Enfermedad de De Quervain. En: Flynn JE. Cirugía de la Mano, $2^{\text {a }}$ edición. Barcelona: Ed Toray SA, 1984: 505-8.

9. Wolfe SW. Enfermedad de De Quervain. En: Green's. Cirugía de la Mano, vol.2 Madrid: Ed Marbán, 2007: 2150-4.

10. Arroyo J, Delgado PJ, Fuentes $A$, et al. Tratamiento quirúrgico de la tenosinovitis estenosante de De Quervain. Patología del Aparato Locomotor, 2007, 5: 88-93.

11. Wetterkamp D, Rieger $\mathrm{H}$, Brug E. Tratamiento quirúrgico de la tenosinovitis estenosante de De Quervain. Tech Quir Ortop Traumatol (edic esp), 1999; 8: 109-14.

12. Rossi C, Cellocco P, Margaritondo E, et al. De Quervain disease in volleyball players. Am J Sports Med, 2005; 33: 4247.

13. Wolf JM, Sturdivant RX, Owens BD. Incidence of de Quervain's Tenosynovitis in a young active population. J Hand Surg Am, 2009; 34: 112-5.

14. Bouchlis G, Bhatia A, Asfazadourian $\mathrm{H}$, et al. Distal insertions of abductor pollicis longus muscle and arthritis of the first carpometacarpal joint in 104 dissections. Ann Chir Main, 1997, 16: 326-37.
15. Elliot BG. Finkelstein's test: a descriptive error that can produce a false positive. J Hand Surg B, 1992; 17: 481-2.

16. Arons MS. De Quervain's release in working women: a report of failures, complications, and associated diagnoses. J. Hand Surg Am, 1987; 12: 540-4.

17. Ilyas AM. Nonsurgical treatment for de Quervain's tenosynovitis. J Hand Surg Am, 2009; 34: 928-9.

18. McKenzie JM. Conservative treatment of de Quervain's disease. Br Med J 1972; 4: 659-60.

19. Harvey FJ, Harvey PM, Horsley MW. De Quervain's disease: surgical or nonsurgical treatment. J Hand Surg Am, 1990;15: 83-7.

20. Witt J, Pess G, Gelberman RH. Treatment of de Quervain's tenosynovitis: a prospective study of the results of injection of steroids and inmobilization in a splint. J Bone Joint Surg Am, 1991; 73: 219-22.

21. Anderson BG, Manthey R, Brouns MC. Treatment of de Quervain's tenosynovitis with corticosteroids. A prospective study of the response to local injection. Arthritis Rheum, 1991; 34: 793-8. 